\title{
APPLICATION OF GRAPH THEORY FOR SCHEDULING OF TRAFFIC LIGHTS \\ SHAKERA TANVEER
}

Department of Mathematics, Government First Grade College Afzalpur, Kalaburagi, Karnataka, India

ABSTRACT
The objective of this paper is to present the application of graph theory of modelling the traffic problems by
representing them in terms of graphs and in particular, the application of compatibility graph corresponding to the
problem, spanning subgraph and circular arc graphs are used to convert the problem to an LP problem. The solution to
the LP problem can be used for traffic management problems with an efficient way to minimize the waiting time of the
traffic participants at junctions, congestions and it has been shown through an example.
KEYWORDS: Graph Theory, LP Problem, Compatibility Graph, Circular Arc Graph, Clique and Mathematical
Modelling

Received: Aug 04, 2017; Accepted: Aug 24, 2017; Published: Oct 21, 2017; Paper Id.: IJMCAROCT20172

\section{INTRODUCTION}

In the present era of the globalized world, Graph theory has gained much attention to almost all the areas of science and other disciplines due to its direct applications of the real life problems. The most common problem of huge cities throughout the world is traffic congestion. It is due to the continuous growth of population and development. Every year new street and highways are built in most of the urban areas to accommodate the growing number of vehicles [1]. The number of increase in vehicles for metropolitan cities led to a time loss of traffic participants, ecological and sound pollution that will in turn increase the traffic accidents. The major hurdles for the growth of many metropolitan cities are the traffic congestion that affects millions of people. New roads can be constructed to improve the situations, but due to its cost and the existing structures, it is difficult in many cases. The current road networks can control the flow of traffic in these situations in a more efficient way [2]. For handling the city traffic in a more proficient way by appropriate traffic management as an alternative of modifying the road infrastructures, a method has been employed in the present paper. An arrow indicates the direction of commuting. The Graph that shows directions is called Digraph while the one without directions is known as a Graph. These features of Graph Theory can easily handle the traffic management problem with mathematical modelling. A distinct type of traffic problem has been solved in this paper by an example of an offering method as an application of circular arc graphs.

The basic and required definitions like circular arc graph, intersection graphs, interval graphs and clique can be found in $[2,3,7 \& 8]$.

\section{PROBLEM STATEMENT}

To install the traffic lights at road junctions such that the traffic must flow smoothly and efficiently at the particular junction is the main objective under study. In the present problem, a specific example is considered and explained how the problem could be modelled and solved. 


\section{AN EXAMPLE}

Figure 1 depicts a four-leg intersection, with five streams of traffic, viz. $a, b, c, d, e$ and are taken from [4].

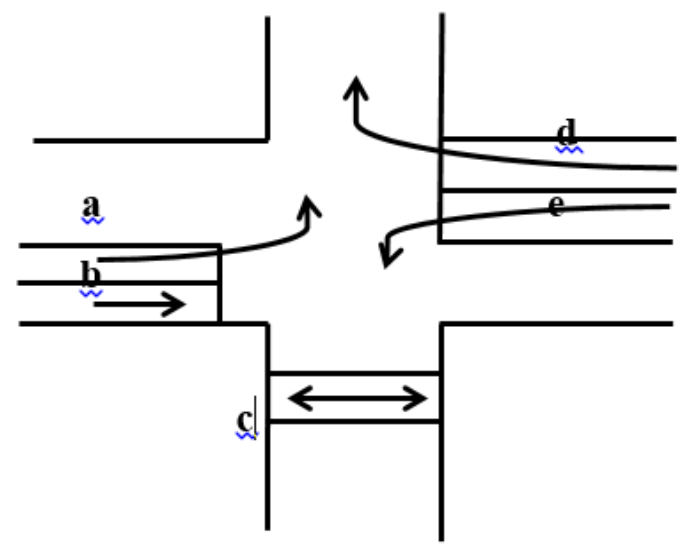

Figure 1: An Intersection with Five Traffic Streams

At an intersection, the vehicles that are approaching towards it prepare themselves to do certain movements, i.e. to drive through, turn left, or turn right at an intersection. Vehicles, which perform these movements, represent a flow component. These arrival flow components are called traffic streams [5]. The traffic streams that moves simultaneously at an intersection without any clash are known as compatible. For example, the traffic streams band is compatible whereas and are non-compatible as shown in Fig. 1. The phasing of lights should be in such a way that the two streams are compatible when the green lights are on for both the streams.We assume that the total time to completeone cycle is 120 second i.e. two minutes during green and red lights. A graph $\mathrm{G}$ is constructed in such a way that its vertex set to consist of the traffic streamsinthe problemand join the two vertices of $G$ by an edgeif and only if, the corresponding streams are compatible.Thus, the obtained graph is the compatibility graph corresponding to the problem under study. Fig. 2 , is the compatibility graph of Figure 1 and are taken from [4].

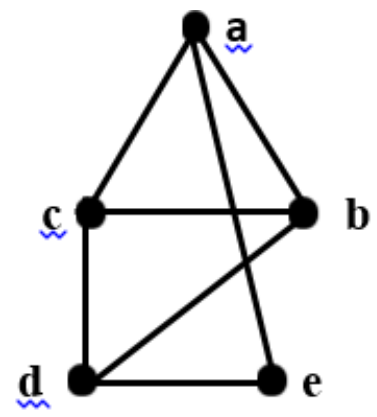

Figure 2: Graph G (Compatibility Graph of Figure 1)

Let us assume a circle and the perimeter of this circle corresponds to the total cycle period, viz. 120 seconds and also consider that an arc of this circle corresponds to the duration when a given traffic stream gets a green light. Therefore, two such arcs of the circle canjoinif and only if the corresponding streams are compatible. The circular arc graph thus obtained may not be the compatibility graph because we are not asserting that two arcs intersect whenever there corresponds to a compatible flow. (Because two streams might not get a green light simultaneously even though they are 
compatible). However, the intersection graph $H$ of this circular arc graph will be a spanning sub graph of the compatibility graph [6]. Here, we have to take all spanning subgraphs of $G$ into account and select from them the spanning sub graph that have the maximal cliques $[3,6]$. The proper groups and $J$ for this exampleisdepicted in Figures $3 \& 4$.

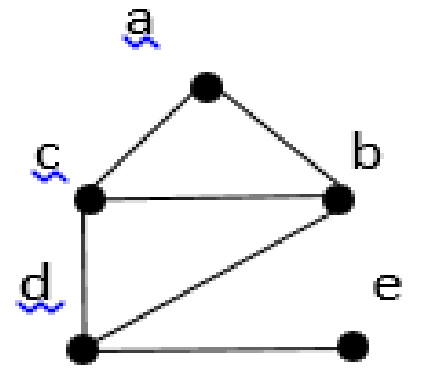

Figure 3: Graph H

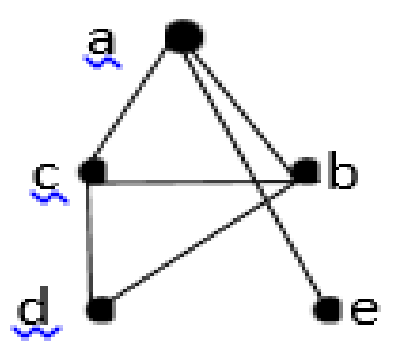

Figure 4: Graph J (Intersection Graphs)

The performance of the phasing may be calculated byminimalizing the total red light time for a traffic cycle is. e. The total waiting time for all the traffic streams during a cycle. For the sake of concreteness, suppose that at the starting time, all lights are red. The maximal clique of $\mathrm{H}$ is $k_{1}=\{a, b, c\}, k_{2}=\{b, c, d\}$ and $k_{3}=\{d, e\}$. To eachclique $k_{i, 1} \leq i \leq$ 3 ,there corresponds a phase during which all the streams in thatclique get a green light. Traffic streams $\mathrm{c}, b$ and a gets a green light in phase 1; in phase 2, $c, d$ and gets a green light; in phase 3, e and d get a green light. Suppose we allocate a duration $d_{i}$ To each phase $k_{i}$. The objective is to find the $d_{i}$ ' $\mathrm{S}(\geq 0)$ and thus to have the minimum total waiting time of the traffic participants. Further, we may consider that for any stream, the minimum green light time is 20 seconds. When the phase $k_{2}$ Gets a green light, then the traffic stream $a$ gets a red light. Hence $a$ 's total red light time is $d_{2}+d_{3}$. Similarly, all the streams b, $c, d$, and e respectively gets a total red light time as $d_{3}, d_{3}, d_{1}$ and $d_{1}+d_{2}$. Therefore, the total red light time for one cycle for all streams is $Z=2 d_{1}+d_{2}+2 d_{3}$. The objective is to minimize $Z$ subject to $d_{i} \geq 0,1 \leq i \leq 3$ and $d_{1} \geq$ 20, $d_{2} \geq 20, d_{3} \geq 20, d_{1}+d_{2} \geq 20, d_{2}+d_{3} \geq 20$ and $d_{1}+d_{2}+d_{3}=120$. The optimum solution to this LP problem is $d_{1}=20, d_{2}=80, d_{3}=20, \min \mathrm{Z}=160$. However, this is not finished yet because there are other possible circular arc graphs. Fig. 4 gives another possible green light arrangement and its corresponding intersection graph. With reference to this graph a similar maximal clique of $\mathrm{J}$ are $k_{1}=\{a, b, c\}, k_{2}=\{b, c, d\}$ and $k_{3}=\{a, e\}$. To each clique $k_{i}, 1 \leq i \leq 3$, there corresponds a phase in which all streams in that clique gets a green light. Traffic streams $a, b$ and $c$ receive a green light in phase 1; in phase 2, $b, c$ and $d$ receive a green light; in phase 3, a and we receive a green light. Suppose we assign to each phase $k_{i}$ A duration $d_{i}$. Again the aim is to find the $d_{i}$ ' $\mathrm{S}(\geq 0)$ in such a way that the total waiting time is minimized. Further, we may assume that the minimum green light time for any stream is 20 seconds. Traffic streams $a$ gets a red light when the phase $k_{2}$ Receive a green light. Hence $a$ 's total red light time is $d_{2}$. Similarly, the total red light time of all streams b, $c, d$, and e respectively are $d_{3}, d_{3}, d_{1}+d_{3}$ And $d_{1}+d_{2}$. Therefore the total red light time of all streams in one cycle is $Z=2 d_{1}+2 d_{2}+3 d_{3}$. The aim is to minimize $Z$ subject to $d_{i} \geq 0,1 \leq i \leq 3, d_{1} \geq 20, d_{2} \geq 20, d_{3} \geq 20, d_{1}+$ $d_{2} \geq 20, d_{1}+d_{3} \geq 20$ and $d_{1}+d_{2}+d_{3}=120$.The optimum solution corresponding to this Linear Programming (LP) problem is $d_{1}=80, d_{2}=20, d_{3}=20$, min $\mathrm{Z}=260$ (in seconds). Hence, by exhausting all possible circular arc graphs and then selecting the least of all the minima so obtained. The least that value relates to this phasing would be the finestphasing of the traffic lights. The least value of the best phasing of the above problem is 160 (in seconds).

\section{CONCLUSIONS}


The present paper gives an insight of the theory of circular arc graphs; here the problem of phasing of traffic lights at junction of four-leg intersection with five traffic streams has been solved by the LP method such that the traffic signals could be usedmoreproficiently to evade the long waiting time at junctions and overcrowding. Two different possible green light arrangements and their corresponding intersection graphs have been plotted to clearly understand the problem and apply it to the practical implementation at various junctions of similar kind and thus could be applied very effectively for day-to-day life.

\section{REFERENCES}

1. D.C. Gazis, (2002), Traffic Science, (John Wiley \& Sons, New York).

2. Darshankumar Dave and Nityangini Jhala, (2014), Application of Graph Theory in Traffic Management, Int. J. Of Engg. And Innovative Tech. Vol. 3 (12) 124-126.

3. Mohsen Hosseini, S. And Oroogi, H., (2009), Phasing of Traffic Lights at a Road Junction, Appl. Math. SCI., 3 (30) 1487-1492

4. Arun Kumar Baruah, (2014), Traffic control problems using graph connectivity, Int. J. Of Comp. App., 86 (11)1-3.

5. Guberinic, S., Senborn, G., Lazic, B. (2008), Optimal Traffic Control UrbanIntersection, CRC Press.

6. Balakrishnan, R., Ranganathan, K. (2012), A textbook of Graph Theory, Second Edition, Springer, New York.

7. Anurag Sharma., Rajeev Pandey., (2011), Optimization of Traffic Signal System, J. Of Int. Academy of Physical sciences., 15 (4) $479-486$.

8. $\quad$ Fred S. Roberts (1978), Graph Theory and its Applications to Problems of Society, Philadelphia. 